\title{
Dosimetric evaluation of the MLCs for irregular shaped radiation fields
}

\author{
Omar Kotb , ${ }^{1}$ Khaled M. Elshahat, ${ }^{2}$ Nabil Mohamed Eldebawi ${ }^{1}$ Nassif Abd \\ Elazim Mansour ${ }^{1}$ \\ ${ }^{1}$ Physics Department- Faculty of Sciences -El Zigzags University - Cairo \\ ${ }^{2}$ Radiation Oncology Dept.- Faculty of Medicine- Al Azhar University-Cairo
}

\begin{abstract}
The three-dimensional conformal radiotherapy (3D-CRT), intensity-modulated radiotherapy (IMRT), and image-guided radiotherapy (IGRT) are the most advanced techniques in radiotherapy, which use irregular fields-using multileaf collimators in a linear accelerator. The accuracy of these techniques depends on dosimetric characteristics of the multileaf collimators. There is an option for optimizing the jaws to the irregular MLC field to reduce the scattered radiation and intra- and inter-leaf radiation leakage beyond the field. In this study, ,80 leaf MLC system has been taken to compare and differentiate their characteristics with 6-MV, and 10-MV photon beams.

The MLC system in Elekta linear accelerator is used as a separate unit, that is, The dosimetric characteristics include dose rates, percentage depth doses, surface dose, dose in the build-up region, penumbra, and width of $50 \%$ dose levels
\end{abstract}

Key words: radiotherapy, multileaf collimator, quality assurance, IMRT.

\section{Introduction}

The goal of radiation therapy is to administer a therapeutic dose of radiation to a target while limiting the side effects caused by delivering the dose to surrounding tissues and vital organs. The ongoing pursuit to achieve an optimal dose distribution has prompted the radiation therapy profession to develop new techniques that incorporate advances in technology. In radiation therapy today, modern techniques that include threedimensional conformal radiation therapy (3D-CRT) and intensity-modulated radiation therapy (IMRT) are routinely used in the treatment of cancers. Compared with 3D-CRT, IMRT is capable of producing dose distributions that conform to the planning treatment volume and deliver a reduced dose to surrounding tissues and vital organs.

This has come with the cost of increased treatment time and a larger volume of normal tissue receiving low radiation doses. Most recently, there has been considerable interest in the rotating gantry IMRT techniques, tomotherapy and volumetric-modulated arc therapy (VMAT). Tomotherapy is a dedicated treatment system that is best described as a combination of a computed tomography scanner and a linear accelerator. In tomotherapy, treatment is delivered using a rotating fan beam. A therapeutic dose is delivered when a patient is translated smoothly through the bore of the machine as its gantry continuously rotates.

Conformity can be achieved through a series of conformal dynamic arcs. In the case of the intensity modulation radiotherapy treatment (IMRT), the change of the field shape is accompanied by the beam on, a certain amount of leaf position error has to be accepted in order to make the treatment deliverable. Therefore, to ensure that the treatment is delivered accurately, it is essential that an efficient and effective quality assurance program can be applied on a routine basis.

The Static test in current paper provide a quantitative information about the leaves locations based on well defined radiation-centre crossed lines, using analysis software for Matrix for Iba company as it is a powerful image analysis tool allowed us to analysis images with high resolution, up fraction of millimeters, and Comparing the measured MLC's A and B values with the expected value listed in MLC file to get the positional errors for each leave over A and B banks. 


\section{Materials and Methods}

All the following equipment was from Elekta Systems,. A linear accelerator Elekta Synergy Platform, equipped with a 80-leaf MLCi, was used for IMRT Therapy. The treatment energy used was $6 \mathrm{MV}$ and $10 \mathrm{MV}$ photons beam. The MLC consists of 40 pairs of leaves of $1 \mathrm{~cm}$ covering fields up to $40 \mathrm{~cm} \times 40$ $\mathrm{cm}$. The MLC is a computer controlled device consists of set of opposing leave pairs moving in the $\mathrm{X}$ jaws direction and fixed below it on two opposing carriages (banks). All these systems were interfaced with Mosaiq ( Radiation Oncology information system) .

ISOCHECK; Isocenter and Iba Blue 3D water phantom and and RW3 slab phantom, is tissueequivalent with a density of $1.04 \mathrm{~g} / \mathrm{cm}^{3}$; its dimensions are $30 \mathrm{~cm} \times 30 \mathrm{~cm} \times 30 \mathrm{~cm}$, , and a Pinpoint ion chamber .All equipment used is produced by Iba (Iba dosimetry company)

\subsection{Dosimetric characteristics of MLC}

In current paper the dosimetric characteristics of multileaf collimators (MLCs) were evaluated for 6MV and 10 -MV photon beams. The percentage depth dose, surface dose, dose in the build-up region, beam profile, flatness, symmetry, and penumbra width were measured using three field-defining methods: (i) 'Jaw only', (ii) 'MLC only', and (iii) 'MLC+Jaw'. Analysis of dose rate shows that the dose rate for 'MLC only' field was higher than that for 'Jaw only" and 'MLC+Jaw' fields in both the energies.

\subsection{Clinical Application for Prostates Case}

To create MLC fields of leaf position with different location that covers a wide range of clinical use, so that separate this wide range of fields to study it individually and take the advantage of MLC repetition in this separation for the study of reproducibility of the MLC.

\subsection{Collimator Rotation Isocenter}

\section{Results and Discussion}

The mechanical rotational isocenter for the collimator axis should be confined to a sphere of $\leq 1.0 \mathrm{~mm}$ radius. Rotation of the collimator from $90^{\circ}$ to $270^{\circ}$ while observing the pointer run-out that verify the worst case run-out meets specification.

Beam Profile for small field Size : for MLci with Jaws and Jaws only

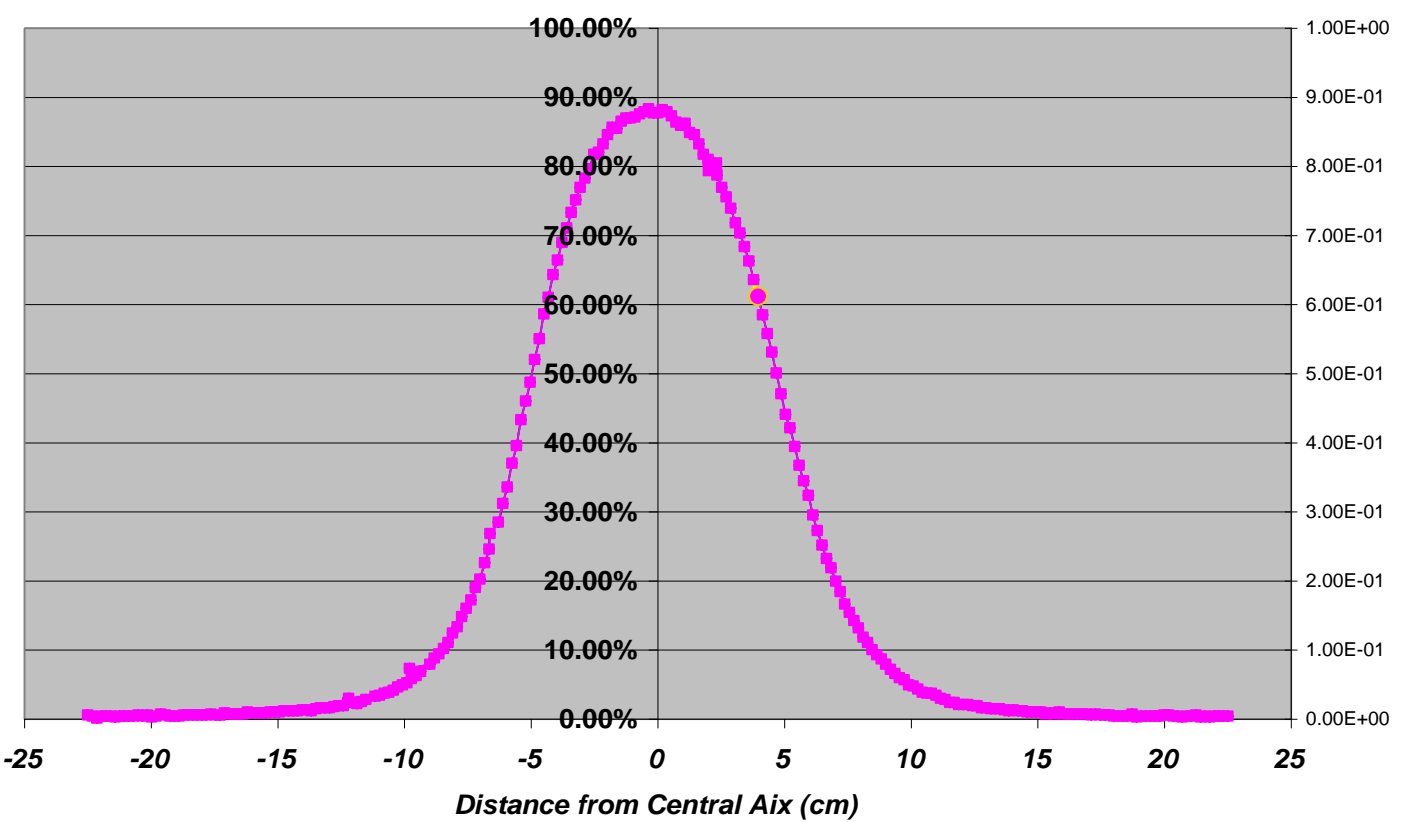

Fig ( 1 ) : Beam Profile for field size $1 \times 1 \mathrm{~cm}^{2}$ for MLCi with Jaws and Jaws Only 


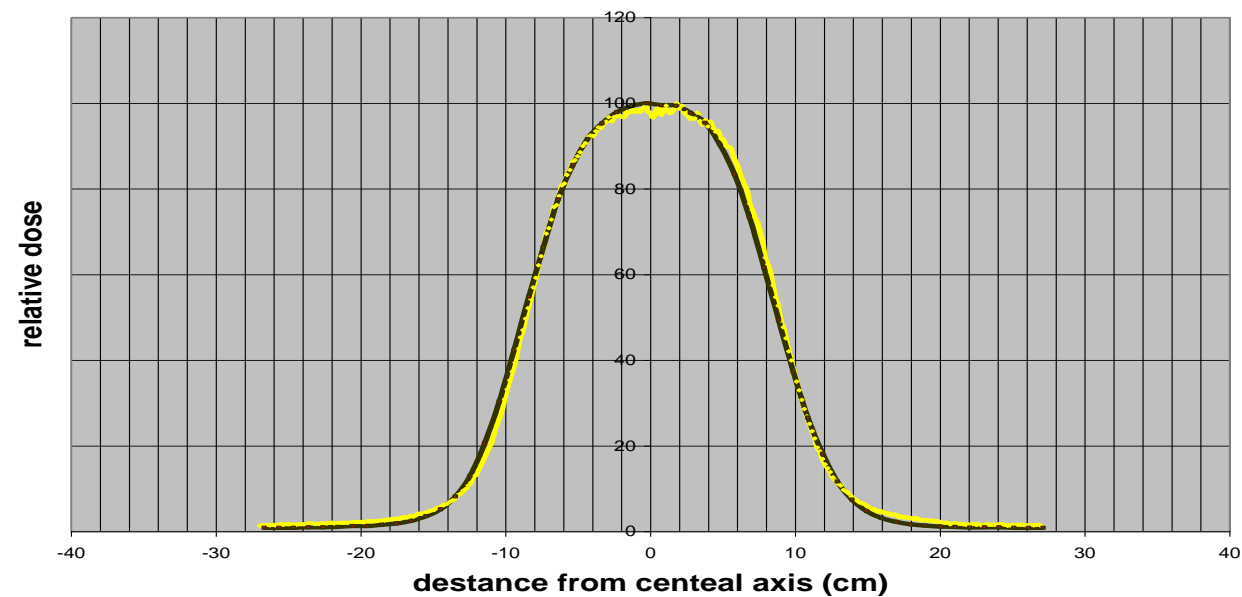

Fig . ( 2) : Beam Profile for field size 1x1 $\mathrm{cm}^{2}$ for MLCi with Jaws and Jaws Only

- The beam profiles were measured using 80 -leaf MLC system, for the square field sizes $1 \times 1 \mathrm{~cm}^{2}, 2 \times 2 \mathrm{~cm}^{2}$, $10 \times 10 \mathrm{~cm}^{2}$, and $20 \times 20 \mathrm{~cm}^{2}$ at $\mathrm{dmax}$ and $10 \mathrm{~cm}$ in the cross-plane orientation for the three field-defining methods mentioned in section D. The flatness and symmetry of the beam profiles were determined for the fields defined above; it was found that the flatness and symmetry were within $2.7 \%$ for both energies. The 'width of $50 \%$ dose level' was measured and analyzed; it was observed that the width of the 'MLC only' field was higher by 2 to $3 \mathrm{~mm}$ for 6-MV and 2 to $2.5 \mathrm{~mm}$ for 10-MV photon when compared with 'Jaw only' and/or 'MLC+ Jaw'

\section{Penumbra for 6MV Photon Beam for MLCi and Jaws}

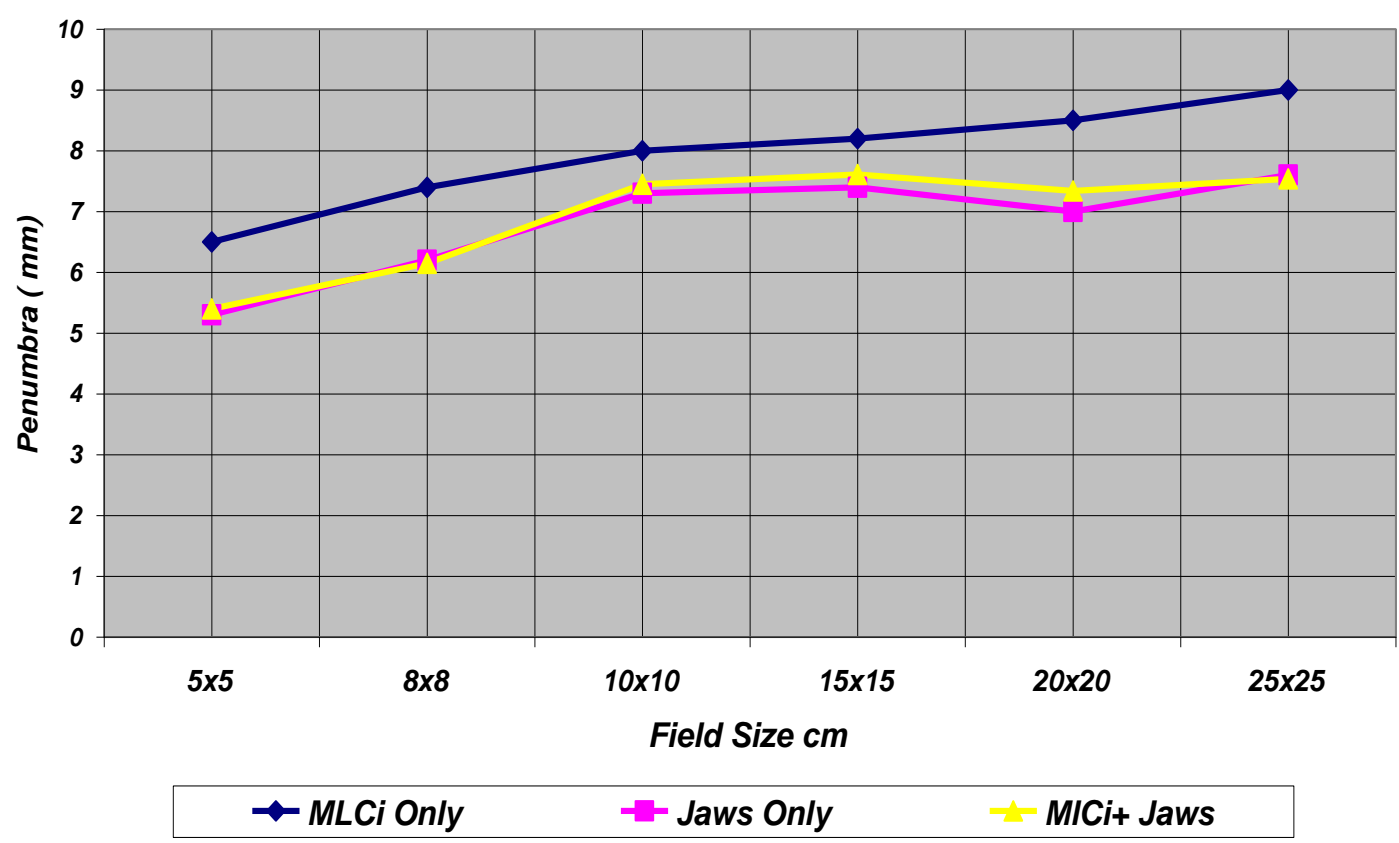

Fig. ( 3) : Penumbra Comparison Between MLCi and Jaws for 6MV photon Beam 


\section{Penumbra for 10 MV photon Beam for MLCi and Jaws}

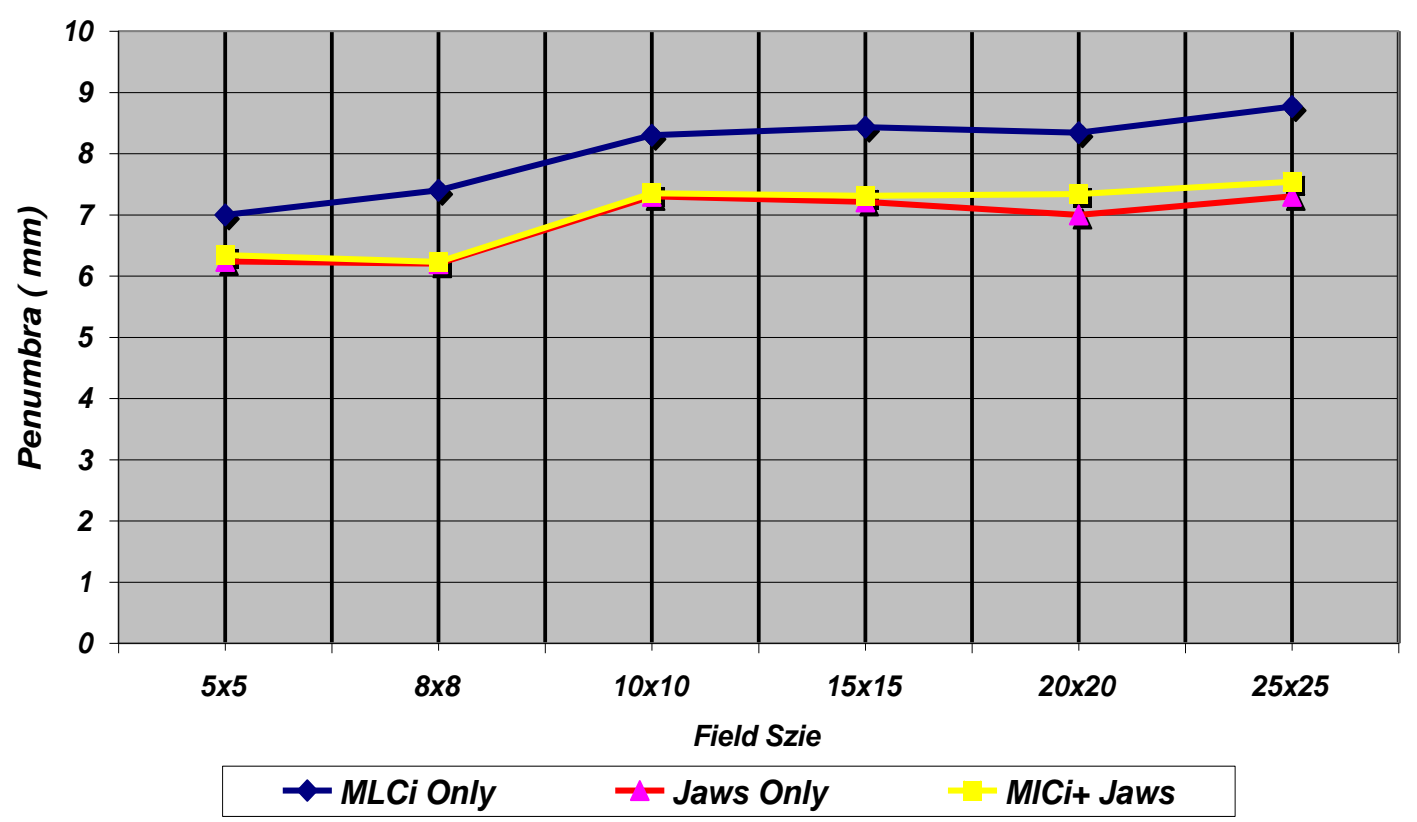

Fig. ( 4) : Penumbra Comparison Between MLCi and Jaws for 10MV photon Beam

- The penumbra ( $80 \%$ to $20 \%$ ) was measured at depth dmax . Penumbra of 'MLC only' field was more than that of 'Jaw only' and 'MLC+ Jaw' by 1.7 to $2.7 \mathrm{~mm}$ for 6-MV and 2.3 to $3 \mathrm{~mm}$ for 10-MV photon beam. Figures 3 and 4 show the penumbra at depth dmax for $6 \mathrm{MV}$ and $10 \mathrm{MV}$ respectively.

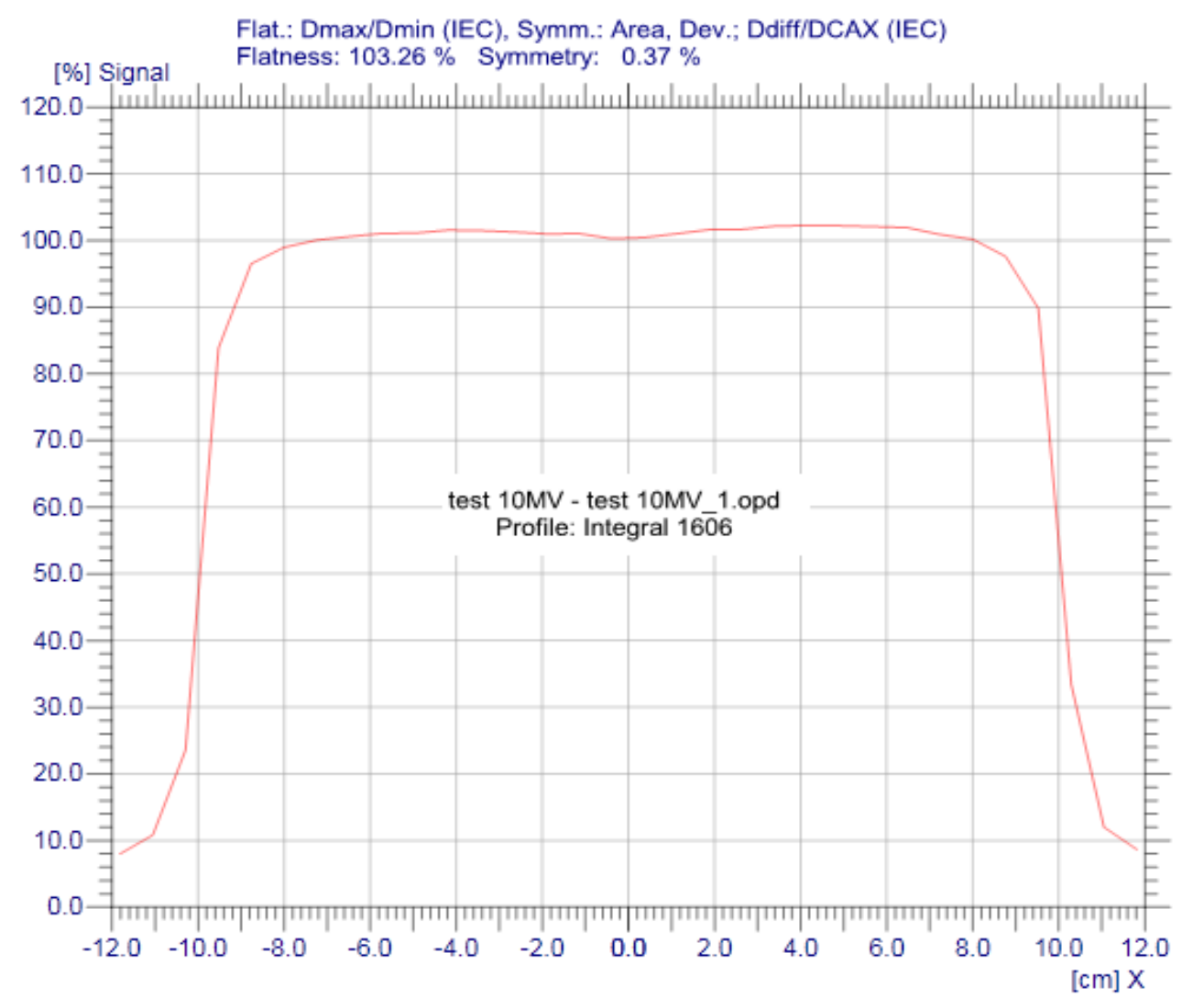

Fig ( 5): Profile for $10 \mathrm{MV}$ X- ray using Iba Matrix to check Energy and flatness before start Measured for Case. 
IMRT is the delivery of radiation to the patient via fields that have non-uniform radiation fluence . The introduction of IMRT creates IMRT improves on the dose distributions achieved using 3D-CRT. 3D-CRT beams are fashioned with tight margins to conform to a target volume. A major limitation of 3D-CRT is that it is unable to account for indentations in the target where critical structures invaginate into the target volume. IMRT addresses this shortcoming of 3D-CRT in that IMRT offers great flexibility in sculpting the dose to complexshaped targets .

The complex shapes achieved using IMRT are made possible in that IMRT considers each radiation beam as multiple rays, or beamlets, and assigns different beam strengths to the individual rays. These beamlets treat small areas of tissue, called voxels, which are a cubic millimetre of space. The beamlets are designated to satisfy the predetermined dose specifications to the tumor site and surrounding normal tissues. By modulating both the number of treatment fields and the intensity within each field, there is a greater control of dose distribution around the target and the dose homogeneity within the target .

\section{IMRT for prostate Case as clinical Application:-}

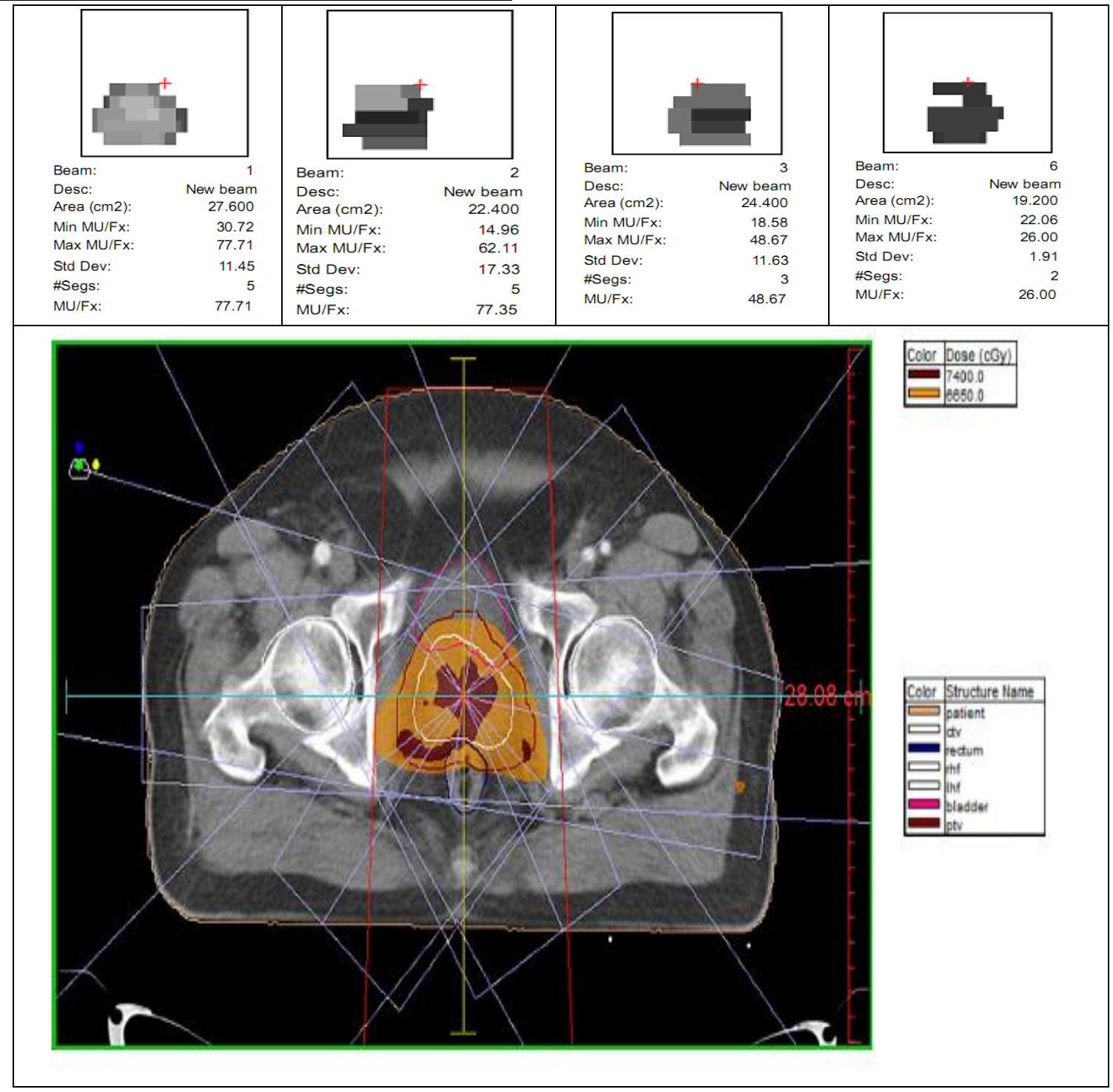

Fig ( 6) : Prostate plan and Fluence for some fields as example 


\section{Comparison between plan and Measurements for Prostate case}

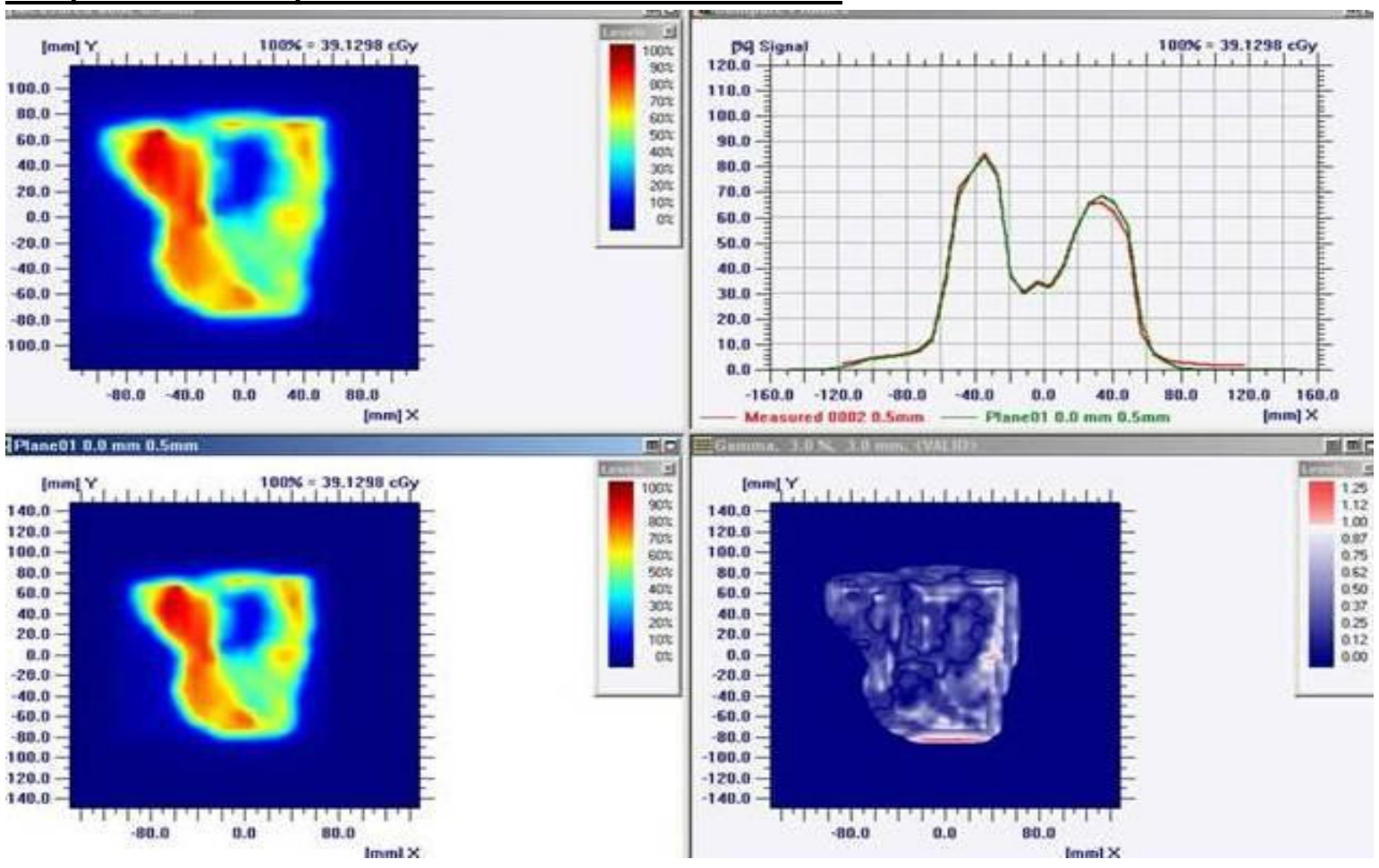

Fig (6): Comparison between Measured and plan data using treatment planning system and Match between tow profiles for Measured (Red color ) and Plan Profile ( Green one )

\section{$\underline{\text { 4. Conclusions }}$}

This protocol was describing the quality assurance (QA) tests on the multileaf collimator (MLC) for IMRT techniques. QA tests were designed and used to simultaneously determine uncertainties associated with MLC for dose delivery errors

The dosimetric characteristics of the Elekta 80-leaf MLCi system were measured, compared, and analyzed using 6-MV and 10-MV photon beams. It was found that its characteristics were quite similar to those of the standard collimator (jaws) system and penumbra. Dose rate for 6-MV and 10-MV photon beams was higher for 'MLC only' field than that for the other two field-defining methods. The PDD comparison shows that the surface dose and dose in the build-up region were more for 'MLC only' fields. Beam profile analysis shows that the flatness and symmetry for both the systems were within $2.6 \%$; the 'width of $50 \%$ dose level' and penumbra were slightly higher for 'MLC only' fields in both energies. The results of this study suggest that standard collimator jaws should be optimized to the irregular MLC field (i.e., MLC+Jaw) to minimize the surface dose, dose rate, penumbra, and dose in the build-up region.

\section{References}

[1]. J. S. Webb $\square$ The physical basis of IMRT and inverse planning Br J Radiol, 76, pp 678-689, (2003).

[2]. J.C. Cash Changing paradigms: intensity modulated radiation therapy Semin Oncol Nurs, 22 pp. $242-248$ (2006).

[3]. M. N. Graves, A. V. Thompson, M. K. Martel, D. L. McShan, and B. A. Fraass, "Calibration and quality assurance for rounded leaf-end MLC systems," Med. Phys. 28, 2227-2233 (2001).

[4]. A. Boyer, P. Biggs, J. Galvin, E. Klein, T. LoSasso, D. Low, K. Mah, and C. Yu, "Basic applications of multileaf collimators," AAPM Radiation Therapy Committee Task Group No. 50 Report No. 72,( 2001).

[5]. J.M. Galvin, W. De NeveIntensity modulating and other radiation therapy devices for dose painting J Clin Oncol, 25 pp. $924-930$ (2007).

[6]. J. M. Galvin et al., "Evaluation of multileaf collimator design for a photon beam,” Int. J. Radiat. Oncol., Biol., Phys. 23, 789-801 (1992). 
[7]. J. M. Galvin, A. R. Smith, and B. Lally, "Characterization of a multi-leaf collimator system,” Int. J. Radiat. Oncol., Biol., Phys. 25, 181-192 (1993).

[8]. T. J. Jordan and P. C. Williams, "The design and performance characteristics of a multileaf collimator," Phys. Med. Biol. 39, 231251 (1994).

[9]. J. Das, G. E. Desobry, S. W. McNeeley, E. C. Cheng, and T. E. Schultheiss, "Beam characteristics of a retrofitted double-focused multileaf collimator," Med. Phys. 25, 1676-1684 (1998).

[10]. A. L. Boyer and S. Li, "Geometric analysis of light-field position of a multileaf collimator with curved ends," Med. Phys. 24, 757762 (1997).

[11]. T.F. Lee, F.M. Fang, P.J. Chao, T.J. Su, L.K. Wang, S.W. Leung Dosimetric comparisons of helical tomotherapy and step-andshoot intensity-modulated radiotherapy in nasopharyngeal carcinoma Radiother Oncol, 89 pp. 89-96 (2008).

[12]. $\square$ G.L. Whitelaw, I. Blasiak-Wal, K. Cooke, C. Usher, N.D. Macdougall, P.N. Plowman A dosimetric comparison between two intensity-modulated radiotherapy techniques: tomotherapy vs dynamic linear accelerator Br J Radiol., 81 pp. 333-340 (2008). 